\title{
Risk of serious spinal adverse events associated with epidural corticosteroid injections in the Medicare population
}

\author{
Efe Eworuke (D) , 'Leah Crisafi, ${ }^{2}$ Jiemin Liao, ${ }^{3}$ Sandia Akhtar, ${ }^{3}$ Martha Van Clief, \\ Judith A Racoosin, ${ }^{1}$ Michael Wernecke, ${ }^{3}$ Thomas E MaCurdy, ${ }^{3}$ Jeffrey A Kelman, ${ }^{4}$ \\ David J Graham ${ }^{1}$
}

- Additional material is published online only. To view, please visit the journal online (http://dx.doi.org/10.1136/ rapm-2020-101778).

${ }^{1}$ Division of Epidemiology, US Food and Drug Administration, Silver Spring, Maryland, USA ${ }^{2}$ Department of Perioperative Medicine, Clinical Center, National Institutes of Health, Bethesda, Maryland, USA ${ }^{3}$ Acumen LLC, Burlingame, California, USA

${ }^{4}$ Centers for Medicare and Medicaid Services Washington DC Office, Washington, District of Columbia, USA

Correspondence to Dr Efe Eworuke, Division of Epidemiology, US Food and Drug Administration, Silver Spring, MD 20993, USA; efe.eworuke@fda.hhs.gov

Received 4 June 2020 Revised 30 October 2020 Accepted 4 November 2020 Published Online First 4 December 2020

\begin{abstract}
Background Epidural corticosteroid injections (ESIs) are widely performed and have an unquantified risk of serious spinal adverse events (SSAEs). We sought to determine the rate of SSAEs following ESI and to compare the rates by spinal level, injection approach and corticosteroid formulation.

Methods We included patients enrolled in Medicare parts A and B who had an ESI between 1 January 2009 and 30 September 2015. We identified potential cases as patients with spine-related diagnoses within 3 days after the first eligible ESI. Event categorization as probable, possible or non-case was based on review of medical records. The rates of probable and possible cases were expressed per 1000000 patients overall, and by spinal level, injection approach and corticosteroid formulation. A score test was used to compare these rates. Results We identified 1355957 eligible ESIs during the study period. Of the 110 potential cases, 43 were selected for medical record review and 11 were categorized as probable, yielding a rate of 8.1 per 1000000 patients ( $95 \% \mathrm{CI} 4.5$ to 14.5). Risk of SSAEs was statistically higher with cervical/thoracic injections (29.4, 95\% Cl 12.5 to 68.8) compared with lumbar/ sacral injections $(5.1,95 \% \mathrm{Cl} 2.3$ to 11.0 ) (p value $0.001)$. Event rates for lumbar/sacral non-transforaminal injections was 8.8 (95\% Cl 4.0 to 19.1). Event rates for particulate $(7.5,95 \% \mathrm{Cl} 3.9$ to 14.2$)$ and non-particulate formulations (13.1, 95\% Cl 3.6 to 47.9) appeared similar ( $p$ value 0.47).

Conclusion Between 2009 and 2015, rates of SSAEs following ESI in the Medicare population were low. Patients receiving cervical/thoracic ESIs were at higher risk of SSAE than those receiving lumbar/sacral ESIs. Event rates were similar for each corticosteroid formulation.
\end{abstract}

\section{INTRODUCTION}

Epidural corticosteroid injections (ESIs) have been widely used in the management of neck, back and radicular pain. Between 1997 and 2014, 90 serious and sometimes fatal neurological events following ESIs were reported to the US Food and Drug Administration (FDA) Adverse Event Reporting System. These included cases of paraplegia, quadriplegia, spinal cord infarction and stroke. ${ }^{1}$ Potential causes of these events included technique-related problems such as epidural hematoma, direct spinal cord injury and embolic cerebral infarction after inadvertent intra-arterial injection. Patient risk factors for these catastrophic events to guide the identification of high-risk patients undergoing an ESI are largely unknown. ${ }^{2-4}$

In May 2014, the FDA required a warning for all injectable corticosteroid product labels stating that 'serious neurologic events, some resulting in death, have been reported with epidural injection of corticosteroids' and that the 'safety and effectiveness of epidural administration of corticosteroids have not been established and corticosteroids are not approved for this use'. ${ }^{5}$ The warning did not distinguish neurological risk by spinal cord level (cervical, thoracic, lumbar or sacral), injection approach to the epidural space (transforaminal or non-transforaminal) or corticosteroid formulation (non-particulate or particulate) because there were no quantitative data indicating a difference in risk by injection approach, spinal cord level or corticosteroid formulation.

In 2015, an expert panel convened by the FDA's Safe Use Initiative reviewed existing evidence regarding neurological complications with ESIs and published recommendations to prevent neurological complications after ESIs. ${ }^{6}$ The report suggested lumbar injections can be as harmful as cervical injections although there have been fewer cases reported, and it noted that some steroid preparations contain particles forming aggregates that may be able to block small terminal arterioles supplying the brain or the spinal cord. The group also noted that more cases have been reported with cervical and lumbar transforaminal approaches than with interlaminar approaches. The expert group concluded catastrophic events with ESIs do occur, but the actual rate is unknown. Further, they acknowledged that the presented clinical considerations were based on the logical opinions of a group of experts, and rigorous scientific research would be need to provide additional evidence.

Therefore, we aimed to estimate the rate of serious spinal adverse events (SSAEs) after ESI in the Medicare population and compared the event rates by spinal cord level, injection approach and corticosteroid formulation. Due to the common occurrence of stroke in the Medicare population, we restricted the study outcome to serious spinal events. 


\section{METHODS}

\section{Data source}

This retrospective cohort study was conducted using fee-forservice Medicare enrollment and claims databases (Enrollment Database and Common Working File). These data are composed of claims data from medical and pharmacy benefits for all Medicare eligible beneficiaries aged 65 years and older, as well as persons under 65 years old who have end-stage renal disease or are disabled. For each enrollee, claims were linked from all settings of care to provide a longitudinal record of each beneficiary's health encounters and diagnoses. ${ }^{7}$

\section{Study population and exposure definitions}

The study sample included all patients, regardless of age, who received at least one ESI between 1 January 2009 and 30 September 2015 and were continuously enrolled in Medicare parts $\mathrm{A}$ and $\mathrm{B}$ for at least 6 months prior to the date of the qualifying ESI. ESIs are covered by Medicare part B and were identified using Current Procedural Terminology (CPT) and Healthcare Common Procedure Coding System (HCPCS) codes (see online supplemental table 1A,B). CPT codes identified the spinal level of the injection (cervical/thoracic or lumbar/ sacral) and approach to the epidural space (transforaminal or non-transforaminal), while the HCPCS codes identified the corticosteroid type (particulate or non-particulate). Thus, we examined four cohorts - transforaminal cervical/thoracic, transforaminal lumbar/sacral, non-transforaminal cervical/thoracic and non-transforaminal lumbar/sacral. To enhance comparability of our study sample and to reduce potential confounding due to additional underlying disorders in patients who receive multiple ESIs, we focused on the first eligible ESI administration for patients with no previous recent ESI administrations. Thus, patients were excluded if they had a CPT code for an ESI or an HCPCS code for a steroid in the 6 months prior to the date of the first eligible ESI administration (index) date. Additionally, we excluded patients with injections qualifying them for multiple cohorts and patients who received HCPCs for both particulate and non-particulate steroids on their index date. To reduce possible exposure misclassification due to the steroid being received for another purpose, we also excluded patients with a claim for an injection at the facet joint (see online supplemental table $1 \mathrm{E}$ for CPT codes) on the same day as the ESI.

\section{Identification and adjudication of SSAEs following epidural administration of corticosteroids}

The outcome of interest was an SSAE. Cases were identified using a three-step process. In the first step, we identified patients admitted to a hospital up to 3 days after an ESI with a diagnosis of quadriplegia, diplegia, monoplegia; spinal cord injury, transverse myelitis, hematoma complicating a procedure, non-traumatic extradural hemorrhage, vascular myelopathy, extradural hemorrhage, subdural hemorrhage, other exploration and decompression of the spinal canal; or an inpatient claim for laminectomy with or without foraminotomy or facetectomy (see online supplemental table $1 \mathrm{C}, \mathrm{D}$ for code definitions). In the second step, for each potential case identified, a chronologial transcript of all inpatient and outpatient claims generated between 30 days before and 30 days after the ESI was independently reviewed by EE and DJG. Cases that EE and DJG determined as unlikely to represent a serious spinal event based on the review of claims were excluded from further review. In the third step, inpatient medical records for the remaining potential cases were obtained and independently reviewed by EE, DJG and LC, with particular attention to history and physical examinations, consultations, imaging studies, operative reports and discharge summaries. Each case was classified as probable (ie, a relationship between ESI and the SSAE is likely); possible (ie, unclear relationship between ESI and SSAE, insufficient evidence to rule out a relationship or possible alternative explanation); or unrelated (ie, the serious outcome was clearly unrelated to the ESI; or there was no SSAE; or no percutaneous ESI was performed). After the classification of cases was completed, EE, DJG, LC, MVC and JR met to compare and discuss the classification of all cases. Cases where the initial classification by EE, DJG and LC differed were discussed in detail, and consensus was reached on the final classification of those cases. The approach to the epidural space and corticosteroid formulation was blinded during the outcome verification process.

\section{Statistical analysis}

We summarized the demographic characteristics of patients who received an eligible ESI by spinal level and approach to epidural space. Standardized mean differences (SMDs) were used to determine the balance in these variables across cohorts, with a value of $\leq 0.10$ indicating a negligible difference between groups. The event rate per 1000000 patients was calculated for the probable cases, and for the probable and possible cases combined. We also calculated event rates and 95\% CIs stratified by spinal level, approach to the epidural space and corticosteroid formulation. Using the score method, ${ }^{89}$ we calculated risk differences and CIs and contrasted the rates by spinal level, approach to the epidural space and corticosteroid formulation. The CPT codes used to identify ESI exposure may not allow researchers to reliably capture the number of injections during an ESI procedure in claims data as physician and institutional billing for the same procedure can make it challenging to determine if multiple claims reflect the total number of injections. Because patients could potentially receive more than one injection, event rates based on the number of patients undergoing ESI could be higher than rates based on the number of injections received. Thus, we conducted a sensitivity analysis where we assumed two injections for patients with additional level CPT codes or a modifier for multiple procedures (modifier '51') (online supplemental table 1A). We recalculated the event rates per 1000000 procedures overall and by spinal level, approach to the epidural space and corticosteroid formulation, where patients with multiple injections were counted twice in the denominator. This study was classified as public health surveillance by the FDA and was exempt from review by its institutional review board. Analyses were performed using R V.3.6.0 (R Foundation for Statistical Computing Vienna, Austria) and SAS V.9.4.

\section{RESULTS}

Over the study period, we identified 1355957 eligible patients. Patients receiving lumbar/sacral-level ESIs ( $n=1 \quad 185$ 686, $87.4 \%$ ) were far more common than those who received cervical/thoracic-level ESIs $(n=170271,12.6 \%)$. Regardless of spinal level, patients who received particulate corticosteroid formulation via the non-transforaminal route were the most predominant (figure 1). Over the study period, the number of patients receiving non-transforaminal particulate injections declined, while those receiving transforaminal non-particulate injections at the lumbar/sacral site increased. While we observed a similar decrease for non-transforaminal particulate cervical/ thoracic injections, there was an increase for non-transforaminal non-particulate injections (figure 1). 
A

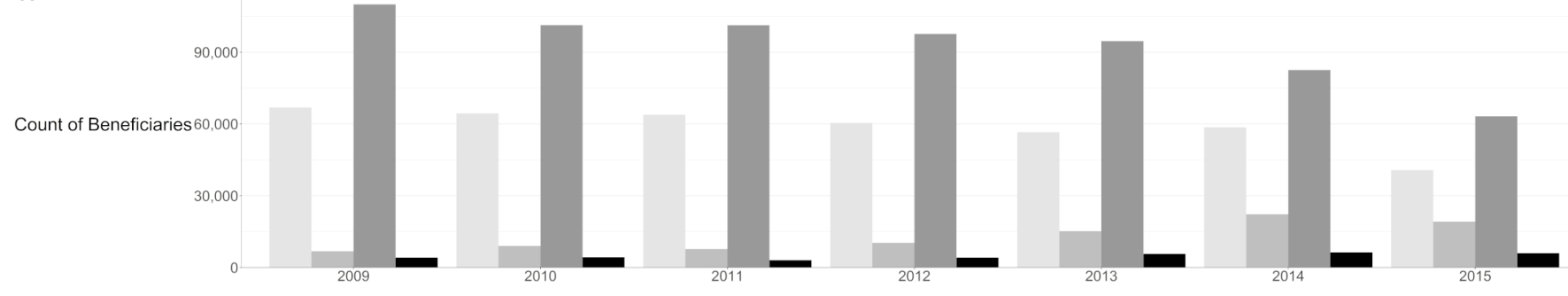

B

$$
30,000
$$$$
2012
$$

2013

2014

2015

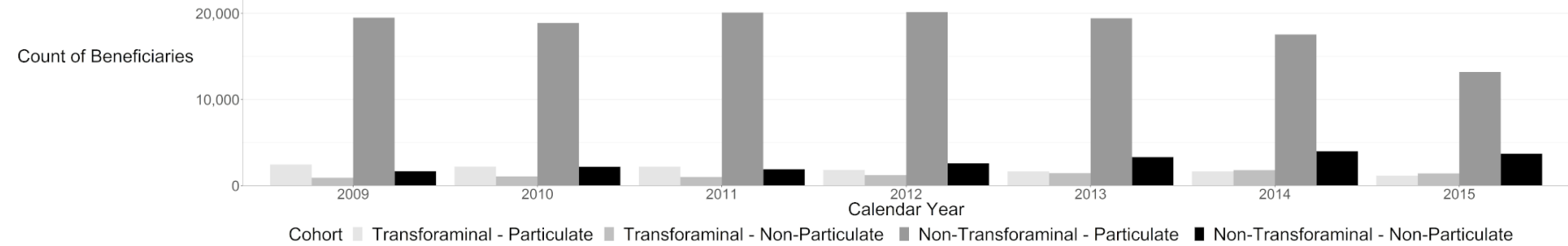

Figure 1 Number of beneficiaries receiving incident lumbar/sacral (A) or cervical/thoracic (B) epidural steroid injections by route of administration and calendar year.

Patients receiving cervical/thoracic injections were slightly younger (mean age 66.8 (transforaminal) and 66.3 years (nontransforaminal)) than those receiving lumbar/sacral injections (mean age 70.8 years (transforaminal) and 71.2 years (nontransforaminal)); more women than men received ESIs with both approaches and both spinal levels (table 1). For cervical/ thoracic injections, demographic characteristics, including age, gender, race, low-income subsidy status and dual MedicareMedicaid eligibility, appeared similar (SMDs $<0.1)$ across transforaminal and non-transforaminal approaches, except for Asian race, where there was a slight imbalance (online supplemental table 2). Likewise, these characteristics were also balanced for the lumbar/sacral injections. Demographic characteristics were also similar between patients who received particulate and nonparticulate injections (online supplemental table 2). Regardless of the spinal level or approach to the epidural space, ESIs were often administered by interventional pain management specialists.

Of 110 potential cases of SSAE identified in step 1 of our case identification process, 43 were selected for medical record retrieval and case classification (figure 2). The medical records of two cases could not be located, leaving 41 potential cases. For 17 potential cases, the ESI code was used to bill for the application of steroids during a spinal surgery and did not represent a stand-alone outpatient ESI procedure. Of the remaining potential cases, 11 were adjudicated as probable, 5 as possible and 8 as unrelated. The probable cases yielded a rate of 8.1 cases per 1000000 patients (95\% CI 4.1 to 14.5 per 1000000 patients). Interventional pain management specialists performed the ESI in 7 of the 11 probable cases and in 2 of the 5 possible cases.

Of the 11 probable cases, 5 received cervical/thoracic and six received lumbar/sacral injections (table 2). The rate of spinal adverse events was statistically higher for cervical/thoracic (29.4 per 1000000 patients (95\% CI 12.5 to 68.8)) than lumbar/sacral injections (5.1 per 1000000 patients $(95 \% \mathrm{CI} 2.3$ to 11.0$)$ ) $(\mathrm{p} \leq 0.001)$ (tables 2 and 3). All six patients receiving lumbar/ sacral injections that resulted in probable cases were performed via the non-transforaminal approach. Of those that received cervical/thoracic injections that resulted in probable cases, three were administered via the non-transforaminal approach and two via the transforaminal approach. The event rate for the transforaminal approach $(90.9,95 \%$ CI 24.9 to 331.4$)$ was numerically but not significantly higher than that for the non-transforaminal approach $(20.2,95 \%$ CI 6.9 to 59.5$)$ for cervical/thoracic injections ( $\mathrm{p}$ value $=0.07$ ) (tables 2 and 3$)$.

For lumbar/sacral injections, we did not observe any events after injections from the transforaminal approach but did observe an event rate of 8.8 per 1000000 patients (95\% CI 4.0 to 19.1) for the non-transforaminal approach $(\mathrm{p}$ value $=0.04)$. Particulate corticosteroid formulations were used in $88.8 \%$ of all ESI injections and were used in 9 of 11 probable cases and in 14 of 16 probable or possible cases (table 1 and online supplemental table 3 ). Across all spinal levels and approaches, the rate of SSAEs was similar with particulate (7.5 per 1000000 patients, 95\% CI 3.9 to 14.2) and non-particulate corticosteroid formulations (13.1 per 1000000 patients, 95\% CI 3.6 to 47.9$)(p=0.47)$. When expressed per procedure in the sensitivity analysis, the 11 probable cases yielded a rate of 6.9 SSAEs per 1000000 procedures (95\% CI 3.8 to 12.3 ). The comparisons across spinal levels and approaches from the sensitivity analyses were consistent with the primary analyses, with the exception of the comparison between transforaminal and non-transforaminal injections overall, which became statistically significant (online supplemental tables 6 and 7).

The clinical presentation of the probable and possible cases are presented in online supplemental table 5). Of the 11 probable cases, epidural hematomas were present in all except one, which was described as an intradural hematoma in the operative note. Patients with cervical/thoracic hematomas following corticosteroid injections presented with a variety of symptoms and signs, including neck pain, upper extremity weakness, hemiparesis or paraparesis. Signs and symptoms of hematomas following lumbar/sacral injections included lower back pain, lower extremity numbness or weakness, saddle anesthesia, neurogenic bladder or fecal incontinence. Of the probable cases, 4 out of the 11 events involved anticoagulant or aspirin use prior to the ESI. In addition, 9 of the 11 probable cases resulted in emergency surgery for spinal cord decompression to evacuate an epidural hematoma. Based on the hospital records reviewed, after hematoma evacuation, four patients had residual neurological deficits 


\section{Original research}

Table 1 Demographic characteristics of patients who were administered an eligible epidural corticosteroid injection ( $N=1355957)$ between 1 January 2009 and 30 September 2015

\begin{tabular}{|c|c|c|c|c|c|c|c|c|}
\hline \multirow[b]{2}{*}{ Demographic variables } & \multicolumn{4}{|c|}{ Cervical/thoracic } & \multicolumn{4}{|c|}{ Lumbar/sacral } \\
\hline & \multicolumn{2}{|c|}{$\begin{array}{l}\text { Transforaminal } \\
(\mathrm{N}=22000)\end{array}$} & \multicolumn{2}{|c|}{$\begin{array}{l}\text { Non-transforaminal } \\
(\mathrm{N}=148271)\end{array}$} & \multicolumn{2}{|c|}{$\begin{array}{l}\text { Transforaminal } \\
(\mathrm{N}=501601)\end{array}$} & \multicolumn{2}{|c|}{$\begin{array}{l}\text { Non-transforaminal } \\
(\mathrm{N}=684085)\end{array}$} \\
\hline Mean age (years) (SD) & \multicolumn{2}{|c|}{$66.8(12.3)$} & \multicolumn{2}{|c|}{$66.3(12.7)$} & \multicolumn{2}{|c|}{70.8 (11.4) } & \multicolumn{2}{|c|}{$71.2(12.0)$} \\
\hline \multicolumn{9}{|l|}{ Age group (years) $(\mathrm{n}, \%)$} \\
\hline 0-44 & 1232 & 5.6 & 9245 & 6.2 & 16467 & 3.3 & 24906 & 3.6 \\
\hline $45-54$ & 2678 & 12.2 & 20284 & 13.7 & 32109 & 6.4 & 46725 & 6.8 \\
\hline $55-64$ & 2977 & 13.5 & 21533 & 14.5 & 44515 & 8.9 & 64128 & 9.4 \\
\hline $65-74$ & 9334 & 42.4 & 58621 & 39.5 & 213318 & 42.5 & 261047 & 38.2 \\
\hline $75-84$ & 4605 & 20.9 & 30018 & 20.2 & 150495 & 30.0 & 211710 & 30.9 \\
\hline $85+$ & 1174 & 5.3 & 8570 & 5.8 & 44697 & 8.9 & 75569 & 11.0 \\
\hline \multicolumn{9}{|l|}{ Gender (n, \%) } \\
\hline Male & 9289 & 42.2 & 60203 & 40.6 & 207251 & 41.3 & 273033 & 39.9 \\
\hline Female & 12711 & 57.8 & 88068 & 59.4 & 294350 & 58.7 & 411052 & 60.1 \\
\hline \multicolumn{9}{|l|}{ Race/ethnicity (n, \%) } \\
\hline White & 18913 & 86.0 & 130780 & 88.2 & 442349 & 88.2 & 608665 & 89.0 \\
\hline Black & 1597 & 7.3 & 11387 & 7.7 & 36174 & 7.2 & 49700 & 7.3 \\
\hline Asian & 554 & 2.5 & 1277 & 0.9 & 5809 & 1.2 & 6103 & 0.9 \\
\hline Hispanic & 411 & 1.9 & 2032 & 1.4 & 7562 & 1.5 & 7859 & 1.1 \\
\hline Other/unknown & 525 & 2.4 & 2795 & 1.8 & 9707 & 1.9 & 11758 & 1.7 \\
\hline Low-income subsidy $(\mathrm{n}, \%)$ & 6048 & 27.5 & 42853 & 28.9 & 100168 & 20.0 & 155668 & 22.8 \\
\hline \multicolumn{9}{|l|}{ Reason for entrance into Medicare* $(n, \%)$} \\
\hline Aged into Medicare & 13166 & 59.8 & 83267 & 56.2 & 366619 & 73.1 & 486626 & 71.1 \\
\hline Disabled into Medicare & 8755 & 39.8 & 64576 & 43.6 & 133695 & 26.7 & 195700 & 28.6 \\
\hline ESRD only & 79 & 0.4 & 428 & 0.3 & 1284 & 0.3 & 1752 & 0.3 \\
\hline Dual eligible (n, \%) & 5039 & 22.9 & 35385 & 23.9 & 83691 & 16.7 & 130053 & 19.0 \\
\hline \multicolumn{9}{|l|}{ Formulation type $(\mathrm{n}, \%)$} \\
\hline Particulate & 13120 & 59.6 & 128843 & 86.9 & 411191 & 82.0 & 650404 & 95.1 \\
\hline Non-particulate & 8880 & 40.4 & 19428 & 13.1 & 90410 & 18.0 & 33681 & 4.9 \\
\hline \multicolumn{9}{|l|}{ Physician specialty $(n, \%)$} \\
\hline Interventional pain management & 10302 & 46.8 & 83756 & 56.5 & 245972 & 49.0 & 297987 & 43.6 \\
\hline Anesthesiology & 2974 & 13.5 & 36840 & 24.9 & 66169 & 13.2 & 175789 & 25.7 \\
\hline Physical medicine and rehabilitation & 4246 & 19.3 & 13388 & 9.0 & 129291 & 25.8 & 71202 & 10.4 \\
\hline Other/unknown & 4478 & 20.4 & 14287 & 9.6 & 60169 & 12.0 & 139107 & 20.3 \\
\hline \multicolumn{9}{|l|}{ Year of injection (n, \%) } \\
\hline 2009 & 3363 & 15.3 & 21194 & 14.3 & 73614 & 14.7 & 114065 & 16.7 \\
\hline 2010 & 3279 & 14.9 & 21101 & 14.2 & 73403 & 14.6 & 105600 & 15.4 \\
\hline 2011 & 3204 & 14.6 & 21989 & 14.8 & 71540 & 14.3 & 104368 & 15.3 \\
\hline 2012 & 3041 & 13.8 & 22746 & 15.3 & 70758 & 14.1 & 101750 & 14.9 \\
\hline 2013 & 3099 & 14.1 & 22758 & 15.3 & 71727 & 14.3 & 100332 & 14.7 \\
\hline 2014 & 3439 & 15.6 & 21562 & 14.5 & 80752 & 16.1 & 88849 & 13.0 \\
\hline 2015 & 2575 & 11.7 & 16921 & 11.4 & 59807 & 11.9 & 69121 & 10.1 \\
\hline
\end{tabular}

${ }^{*}$ There were a small number of beneficiaries for whom the reason for entrance into Medicare was missing ESRD, end stage renal disease.

that included weakness or dysfunction of the bowel or bladder (online supplemental table 5).

\section{DISCUSSION}

To our knowledge, this is the first large observational study to quantify the rate of SSAEs following ESI and to compare the rates by spinal level, injection approach and corticosteroid formulation. Our study suggests that these events are rare in a large, predominantly older US population. All spinal adverse events identified in our study were clinically significant hematomas. Although uncommon, hematomas are a known risk of ESI and result from damage to the venous plexi during needle placement. Of note, several reports ${ }^{10-17}$ of serious injuries after ESI have also described hematomas as a possible etiology for spinal cord injury following ESI. The onset of symptoms following spinal injury is also consistent with previous reports. Symptoms could begin within a few minutes to an hour but often took 6-48 hours to develop. Three of the identified probable cases were taking warfarin; two others used aspirin or other antiplatelet agents. The occurrence of hematoma after ESI in patients on anticoagulant or antiplatelet medications is consistent with previous reports. $^{2} 131418$ We were unable to confirm if restriction of these medications occurred prior to the ESI because outpatient records were not reviewed for this study. This observation highlights the importance of considering the risks and benefits of withholding anticoagulant and antiplatelet medications, noting 


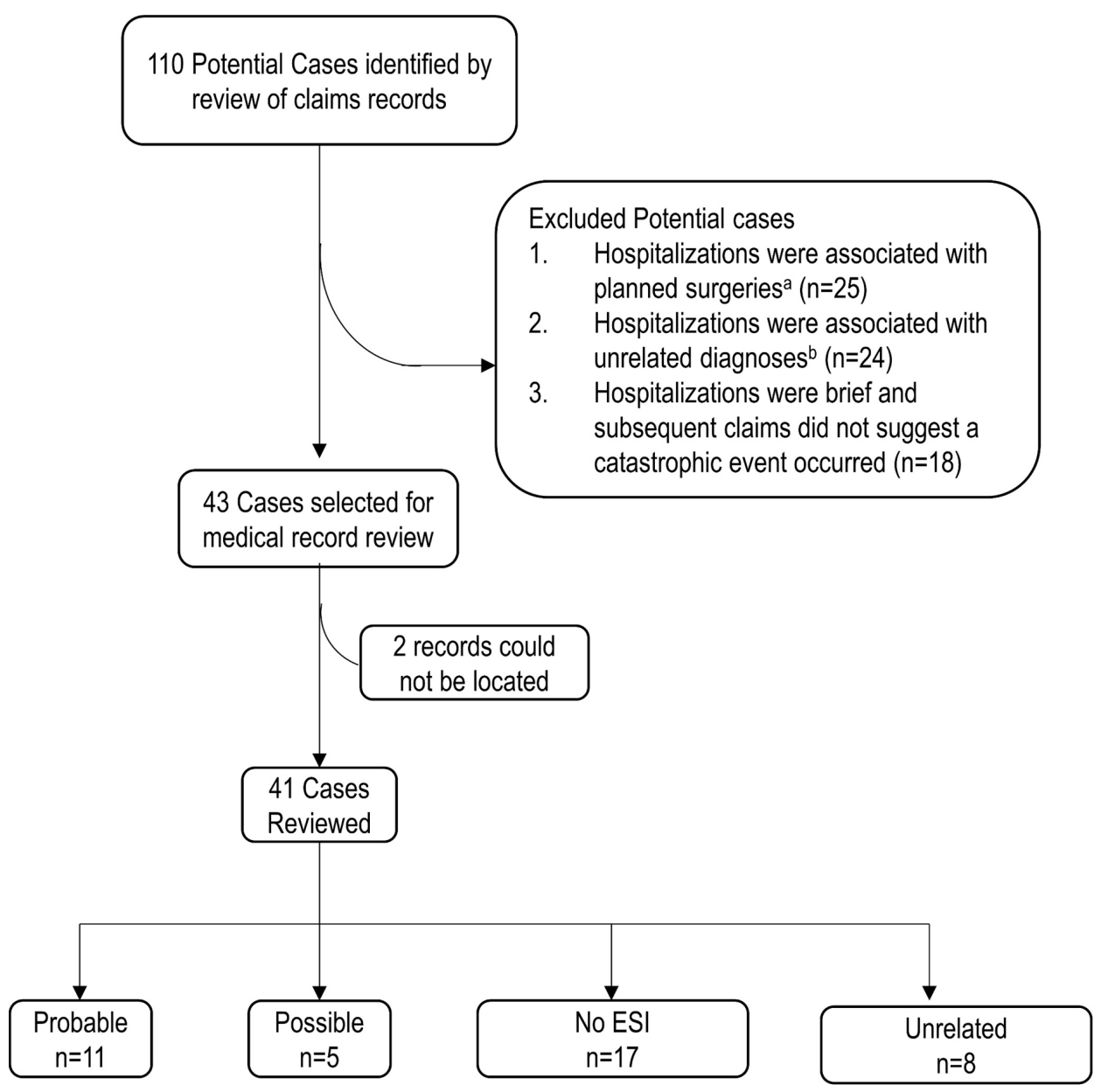

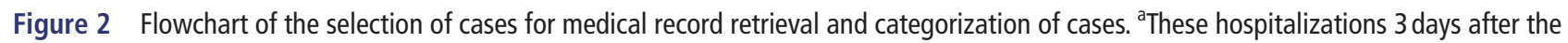
injection were determined to be associated with planned surgeries due to presence of billing codes for preoperative blood work and preoperative cardiovascular examination. bunrelated diagnoses included cardiac-related diagnoses, falls or cerebral diagnoses. ESI, epidural corticosteroid injection.

that withholding these medications for a period of time prior to an ESI presents its own risk (eg, stroke or thromboembolic events). ${ }^{19}$

Our study revealed the following findings about the characteristics of the ESIs and their relationship to the SSAE outcome. First, the risk associated with cervical/thoracic epidural steroid injections was higher than that with lumbar/sacral injections. This may relate to a higher rate of traumatic needle insertion at the cervical/thoracic levels as compared with the lumbar/sacral levels. ${ }^{20}$ An anatomical feature of the cervical/thoracic spinal level is the smaller size of the epidural space that may increase the likelihood of a patient developing clinical symptoms if there is bleeding in the epidural space. ${ }^{6}$ Our data also showed that over time, the number of non-particulate formulations used for cervical/thoracic injections via the transforaminal approach increased slightly with a corresponding decrease in particulate formulations for this approach. This observed trend for particulate formulations in cervical/thoracic transforaminal injections is consistent with recommendations of a panel conveyed by the FDA's Safe Use Initiative, ${ }^{6}$ as well as recommendations from the WIP Benelux Working Group. ${ }^{21}$ Second, while there was no difference in risk when comparing transforaminal versus nontransforaminal approaches across all ESIs in our primary analysis, our data suggested that the transforaminal approach might

Table 2 Rate of serious spinal adverse events (probable cases per 1000000 patients) reported by route of administration and anatomical site of injection

\begin{tabular}{|c|c|c|c|c|c|c|c|c|c|}
\hline \multirow[b]{2}{*}{ Spinal level } & \multicolumn{3}{|l|}{ Total } & \multicolumn{3}{|c|}{ Transforaminal $(\mathrm{N}=523601)$} & \multicolumn{3}{|c|}{ Non-transforaminal $(\mathrm{N}=832356)$} \\
\hline & Events (n) & $\begin{array}{l}\text { Eligible } \\
\text { patients (n) }\end{array}$ & Rate $(95 \% \mathrm{Cl})$ & Events ( $\mathrm{n}$ ) & $\begin{array}{l}\text { Eligible } \\
\text { patients (n) }\end{array}$ & Rate $(95 \% \mathrm{Cl})$ & Events (n) & $\begin{array}{l}\text { Eligible } \\
\text { patients }\end{array}$ & Rate $(95 \% \mathrm{Cl})$ \\
\hline Total & 11 & 1355957 & 8.1 (4.5 to 14.5$)$ & 2 & 523601 & $3.8(1.1$ to 13.9$)$ & 9 & 832356 & 10.8 (5.7 to 20.6$)$ \\
\hline Cervical/thoracic & & & & 2 & 22000 & 90.9 (24.9 to 331.4) & 3 & 148271 & 20.2 (6.9 to 59.5$)$ \\
\hline Particulate & \multirow{2}{*}{5} & \multirow{2}{*}{170271} & \multirow{2}{*}{29.4 (12.5 to 68.8$)$} & 1 & 13120 & 76.2 (13.5 to 431.7$)$ & 2 & 128843 & 15.5 (4.3 to 56.6$)$ \\
\hline Non-particulate & & & & 1 & 8880 & $112.6(19.9$ to 637.7$)$ & 1 & 19428 & 51.5 (9.1 to 291.5$)$ \\
\hline Lumbar/sacral & & & & 0 & 501601 & $0.0(0.0$ to 7.7$)$ & 6 & 684085 & $8.8(4.0$ to 19.1$)$ \\
\hline Particulate & \multirow{2}{*}{6} & \multirow{2}{*}{1185686} & \multirow{2}{*}{$5.1(2.3$ to 11.0$)$} & 0 & 411191 & - & 6 & 650404 & 9.2 (4.2 to 20.1$)$ \\
\hline Non-particulate & & & & 0 & 90410 & - & 0 & 33681 & - \\
\hline
\end{tabular}


Table 3 Risk differences and score test $p$ values for event rate (probable cases per 1000000 patients) comparisons

\begin{tabular}{llc}
\hline Event rate comparisons & Risk difference $(\mathrm{Cl})$ & P value \\
\hline Cervical/thoracic versus lumbar/sacral & $24.3(6.7$ to 63.8$)$ & $<0.001$ \\
\hline Transforaminal versus non-transforaminal & $-7.0(-17.3$ to 4.0$)$ & 0.16 \\
$\begin{array}{l}\text { Cervical/thoracic (transforaminal vs non- } \\
\text { transforaminal) }\end{array}$ & $70.7(-3.8$ to 311.6$)$ & 0.07 \\
$\begin{array}{l}\text { Lumbar/sacral (transforaminal vs non- } \\
\text { transforaminal) }\end{array}$ & $-8.8(-19.1$ to -1.1$)$ & 0.04 \\
\hline
\end{tabular}

carry a higher risk for cervical/thoracic injections, while risk may be greater with the non-transforaminal approach for lumbar/ sacral injections. The low event counts could have affected the precision of comparison of the risk estimates and impedes our interpretation of this possible effect modification. The higher risk of SSAEs with the non-transforaminal than transforaminal approach overall observed in the sensitivity analyses was largely driven by the difference in rates between approaches for the lumbar/sacral injections. It is possible that since traumatic needle insertion is more common with the non-transforaminal (interlaminar) approach, there is an increased risk of hematoma formation. Third, although we observed similar rates of SSAEs with particulate and non-particulate injections, most cases involved particulate formulations. The predominance of particulate injections among cases of serious spinal cord adverse events reflects the overall predominance of particulate injections in the US Medicare population during the study period.

Our study had unique strengths. It used a claims database that was nationally representative and captured a large number of patients who received ESIs. This allowed for the evaluation of adverse spinal events following ESI. Our study also assessed a range of clinically relevant spinal events. A relationship between the events and ESI was also confirmed by medical record abstraction, increasing the validity of the cases identified in the database.

Our study also had several limitations. Although the 3-day time window allowed us to identify plausible adverse events associated with the ESI, we were unable to capture complications of ESI that may take a longer time to develop, such as epidural abscesses. Our study presents unadjusted comparisons of event rates by spinal level, injection approach and corticosteroid formulation. Low event counts hindered the estimation of adjusted event rates. Although we are not aware of patient-level factors impacting both the type of injection (spinal level, route of approach and steroid formulation) received and risk of serious spinal adverse outcomes following administration of ESIs, we cannot rule out the role of confounders. A non-patient-related risk factor for spinal cord events associated with ESIs discussed in the literature is the non-use of live fluoroscopy and digital subtraction angiography to guide needle placement and to help avoid intravascular penetration during these interventions. We were unable to examine specific imaging techniques due to the change in CPT coding in the claims data. Prior to 2011, the billing for imaging required separate CPT codes (77003 for fluoroscopic guidance and 77012 for CT guidance) in addition to the CPT code for the ESI administration. From 2011 onwards, fluoroscopic guidance was bundled into the CPT codes for ESIs, making it difficult to identify which ESIs were administered with imaging guidance after 2011. Another limitation of our study was our inability to distinguish between approaches used for non-transforaminal ESIs that differ greatly in technique (eg, intralaminar vs caudal approach) because the billing code is the same. In addition, the billing code does not report the specific spinal level (ie, cervical 1-8 (C1-C8), thoracic 1-12 (T1-T12), lumbar 1-5 (L1-L5) or sacral (S1-S5)), so we were also unable to determine the influence of the specific spinal level of the ESI administered on the risk of serious spinal cord adverse events. Moreover, as all the epidural injections evaluated involved steroids, we were unable to disentangle the impact of the needle insertion itself from the impact of the injected steroid on the rate of SSAEs. Because our study included only the first eligible ESI, our incidence rates may not be generalizable to patients who have repeat ESIs performed for the same indication in rapid succession, as is the clinical practice in some settings. Lastly, due to the limitations of the claims data, we were not able to accurately report the event rates per injection. In our sensitivity analyses, we attempted to examine the influence of multiple injections by accounting for the potential for multiple-level injections. Although our findings regarding comparative risks by spinal level and route of administration were largely consistent with the primary analysis, the event rates per injection may be lower than our reported rates per patient or procedure if patients receive more than two injections in a single administration.

\section{CONCLUSION}

In this large national sample of Medicare patients, SSAEs were rare. However, cervical/thoracic ESIs were associated with a significantly higher risk of these events compared with lumbar/ sacral ESIs. Our data also suggested that transforaminal approach might carry a higher risk of SSAEs for cervical/thoracic ESIs, while risk may be greater for the non-transforaminal approach for lumbar/sacral ESIs. Event rates for particulate and for nonparticulate corticosteroid formulations were similar.

Contributors All authors contributed to the conception of the study design, interpretation of data, revision of the manuscript critically for important intellectual content and gave the final approval of the version published.

Funding This study was funded by the US. Food and Drug Administration (FDA) through an Inter-agency agreement with the Centers for Medicare \& Medicaid Services.

Disclaimer The views expressed in this publication are those of the authors and do not necessarily reflect the official policy of the Food and Drug Administration, the Centers for Medicare \& Medicaid Services, or the Department of Health and Human Services.

Competing interests None declared.

Patient consent for publication Not required.

Provenance and peer review Not commissioned; externally peer reviewed.

Data availability statement Data may be obtained from a third party and are not publicly available. The data used for this study are not publicly available.

ORCID iD

Efe Eworuke http://orcid.org/0000-0001-8971-9748

\section{REFERENCES}

1 Anesthetic and Analgesic Drug Products Advisory Committee. FDA presentations for the 2014 meeting materials, 2017. Available: https://wayback.archive-it.org/7993/ 20170403223629/https://www.fda.gov/AdvisoryCommittees/CommitteesMeetingMat erials/Drugs/AnestheticAndAnalgesicDrugProductsAdvisoryCommittee/ucm390304. htm [Accessed 6 Jun 2019].

2 Schreiber AL, McDonald BP, Kia F, et al. Cervical epidural steroid injections and spinal cord injuries. Spine J 2016;16:1163-6.

3 Malhotra G, Abbasi A, Rhee M. Complications of transforaminal cervical epidural steroid injections. Spine 2009;34:731-9.

4 Abbasi A, Malhotra G, Malanga G, et al. Complications of interlaminar cervical epidural steroid injections: a review of the literature. Spine 2007;32:2144-51.

5 FDA drug safety communication: FDA requires label changes to warn of rare but serious neurologic problems after epidural corticosteroid injections for pain, 2014 Available: http://www.fda.gov/Drugs/DrugSafety/ucm394280.htm [Accessed 6 Jun 2019]. 


\section{Original research}

6 Rathmell JP, Benzon HT, Dreyfuss $\mathrm{P}$, et al. Safeguards to prevent neurologic complications after epidural steroid injections: consensus opinions from a multidisciplinary Working group and national organizations. Anesthesiology 2015;122:974-84.

7 Houstoun M, Reichman ME, Graham DJ, et al. Use of an active surveillance system by the FDA to observe patterns of quinine sulfate use and adverse hematologic outcomes in CMS Medicare data. Pharmacoepidemiol Drug Saf 2014;23:911-7.

8 Agresti A, Coull BA. Approximate is Better than "Exact" for Interval Estimation of Binomial Proportions. The American Statistician 1998;52:119-26.

9 Miettinen 0 , Nurminen M. Comparative analysis of two rates. Stat Med 1985:4:213-26.

10 Stoll A, Sanchez M. Epidural hematoma after epidural block: implications for its use in pain management. Surg Neurol 2002;57:235-40.

11 Reitman CA, Watters W. Subdural hematoma after cervical epidural steroid injection. Spine 2002:27:E174-6.

12 Litz RJ, Hübler M, Koch T, et al. Spinal-Epidural hematoma following epidural anesthesia in the presence of antiplatelet and heparin therapy. Anesthesiology 2001;95:1031-3.

13 Onishchuk JL, Carlsson C. Epidural hematoma associated with epidural anesthesia: complications of anticoagulant therapy. Anesthesiology 1992;77:1221-3.
14 Dickman CA, Shedd SA, Spetzler RF, et al. Spinal epidural hematoma associated with epidural anesthesia: complications of systemic heparinization in patients receiving peripheral vascular thrombolytic therapy. Anesthesiology 1990;72:947-50.

15 Williams KN, Jackowski A, Evans PJ. Epidural haematoma requiring surgical decompression following repeated cervical epidural steroid injections for chronic pain. Pain 1990;42:197-9.

16 Helperin SW, Cohen DD. Hematoma following epidural anesthesia: report of a case. Anesthesiology 1971;35:641-4.

17 Butler AB, Green CD. Haematoma following epidural anaesthesia. Can Anaesth Soc J 1970;17:635-9.

18 Benzon HT, Wong HY, Siddiqui T, et al. Caution in performing epidural injections in patients on several antiplatelet drugs. Anesthesiology 1999;91:1558-9.

19 Smith CC, Schneider B, McCormick ZL, et al. Risks and benefits of ceasing or continuing anticoagulant medication for image-guided procedures for spine pain: a systematic review. Pain Med 2018;19:438-48.

20 Rathmell JP, Michna E, Fitzgibbon DR, et al. Injury and liability associated with cervical procedures for chronic pain. Anesthesiology 2011;114:918-26.

21 Van Boxem K, Rijsdijk M, Hans G, et al. Safe use of epidural corticosteroid injections: recommendations of the WIP Benelux work group. Pain Pract 2019;19:61-92. 